\title{
Management of Patients with Asymptomatic Carotid Stenosis May Need to Be Individualized: A Multidisciplinary Call for Action
}

\author{
Anne L. Abbott ${ }^{\mathrm{a}, \mathrm{b}}$ \\ ${ }^{a}$ Department of Neuroscience, Central Clinical School, Monash University, Melbourne, Australia \\ ${ }^{b}$ Knox Private Hospital, Wantirna, Australia
}

\section{Dear Sir:}

In a recent Journal of Stroke article, Paraskevas et al. ${ }^{1}$ called for action in using stroke prevention medical intervention (lifestyle coaching and medications) and procedures (such as carotid endarterectomy [CEA] and carotid artery stenting [CAS]). The authors' message was that management of asymptomatic carotid stenosis patients may need to be indivisualized. However, the authors should have gone further and concluded that such management should be indivisualized, given it is clear that patient outcomes vary greatly depending on both patient and intervention factors. ${ }^{2}$ Moreover, the authors omitted full disclosure regarding the most important treatment consideration, the likelihood of patient benefit from a particular treatment.

With respect to asymptomatic carotid stenosis patients, it is only highly selected men aged $<75$ to 80 years with a life expectancy of $\geq 3$ to 5 years and considered at low or average surgical risk who have ever been shown to benefit, overall, from CEA compared to medical intervention alone. ${ }^{2}$ There has never been conclusive randomized trial evidence that women with asymptomatic carotid stenosis so benefit. Women did not benefit from CEA in the Asymptomatic Carotid Atherosclerosis Study (ACAS), which remains the largest randomized trial of asymptomatic carotid stenosis patients comparing stroke rate with medical intervention alone versus with additional CEA. ${ }^{2,3}$ Women coming closest to an overall benefit from early CEA compared to delayed CEA in the Asymptomatic Carotid Surgery Trial (ACST-1) were aged $<75$ years. However, in contrast to Paraskevas et al., the result just failed to reach statistical significance. ${ }^{4}$
Randomized trial evidence is commonly taken as best ('level 1 1) evidence for guiding routine practice treatment decisions. Therefore, it is particularly important to convey to asymptomatic carotid stenosis patients that there is no current randomized trial evidence of them benefiting from CEA. Quality studies have demonstrated that the average annual ipsilateral stroke rate in patients with $\geq 50 \%$ asymptomatic carotid stenosis has fallen by $\geq 58 \%$ over the last $3-4$ decades and since ACAS and the ACST trials were commenced. ${ }^{2}$ The latest average annual ipsilateral stroke rates approximate only $1.0 \%$ with medical intervention alone, similar to, or lower than, with CEA (or CAS) in previous randomized trials. ${ }^{2}$ Medical intervention has improved again since these studies. Definitions of risk factors and treatment goals continue to improve. Even lower stroke rates are now expected without a carotid procedure. No more than about 5.5\% of asymptomatic carotid stenosis patients could now benefit from a carotid artery procedure, and this assumes that the procedural stroke and death rate is always zero. ${ }^{2}$

As acknowledged by Paraskevas et al., many factors have been proposed as identifying asymptomatic carotid stenosis patients at high enough ipsilateral stroke rate, despite medical intervention, to justify CEA. ${ }^{5}$ However, it is important to convey to patients that such markers (particularly when not clearly defined) are too common to identify the very small proportion of patients who could now possibly benefit from CEA and associated ipsilateral strokes rates (where measured) are generally low. ${ }^{2}$ All previous risk stratification studies over-estimate potential CEA benefit because they did not involve current optimal medical intervention. Further, no 'high stroke risk markers' have been tested in randomized CEA trials. It was excellent to

Copyright (C) 2022 Korean Stroke Society

This is an Open Access article distributed under the terms of the Creative Commons Attribution Non-Commercial License (http://creativecommons.org/licenses/by-nc/4.0/) which permits unrestricted non-commercial use, distribution, and reproduction in any medium, provided the original work is properly cited. 
see Paraskevas et al. concluding that non-randomized trial evidence should also be considered for guidelines. Risk stratification studies using current best practice medical intervention alone are the priority for asymptomatic carotid stenosis patients, rather than randomized procedural trials. For example, if ipsilateral stroke rates are sufficiently low, or non-stroke death rates sufficiently high, with current optimal medical intervention, then clearly a carotid artery procedure is not indicated. ${ }^{2}$

Meanwhile, Paraskevas et al. forgot to explain that patients are more likely to suffer peri-procedural stroke, death or myocardial infarction if they undergo CAS compared to CEA. ${ }^{2}$ Individual randomized trials of CAS versus CEA involving asymptomatic carotid stenosis patients were underpowered to compare peri-procedural stroke or death rates, but they showed a trend towards nearly twice as many peri-procedural events with CAS. ${ }^{2}$ These trials include ACST- $2 .{ }^{6}$ The excess peri-procedural harm with CAS in asymptomatic patients reached statistical significance in a randomized trial meta-analysis, and is consistent with the statistically significant increased risk of peri-procedural stroke with CAS in randomized trials of symptomatic patients and in registries. ${ }^{2,7}$ In randomized trials of symptomatic with/without asymptomatic carotid stenosis patients, the long-term stroke rate was also higher with CAS.,9 Given that rates of new stroke after the peri-procedural period are similar with CAS and CEA, evidently patients are more likely to suffer peri-procedural stroke from CAS and live long-term with that stroke.

Paraskevas et al. called for action. To clarify, action is required to:

(1) Acknowledge subgroups where the peri-procedural stroke or death rate is unacceptably high and/or those unlikely to live long enough to benefit from a carotid artery 'revascularization' procedure. These subgroups include the elderly, frail, those with significant comorbidities and those having procedures in low volume centers.'

(2) Acknowledge there is no current randomized evidence of carotid procedural benefit in asymptomatic carotid stenosis patients and there may never be given how low stroke rates are with medical intervention alone.

(3) Give-up the generally accepted routine procedural approach for asymptomatic carotid stenosis so we can perform the required research and provide only currently proven effective therapy. Stroke risk stratification studies using current optimal medical intervention alone are required. However, higher stroke rate does not mean procedural benefit. Only those with sufficiently high residual ipsilateral stroke rate should be considered for procedural trials. This residual rate should be $\geq 3 \%$ to $4 \%$ given the
ACAS experience, where the average annual ipsilateral stroke rate with medical intervention alone was 2.3\%, and to buffer against net patient harm- given that routine practice peri-procedural stroke and death rates are often, if not usually, higher than in randomized trials. ${ }^{10}$ Some cite reasons such as clinician's opinion, poor patient compliance, not meeting current standards of risk factor goals or improved procedural outcomes as procedural justification. This is insufficient. Consistent with evidence-based medicine and first doing no harm, procedural benefit in randomized trials should be shown. This is especially important because asymptomatic carotid stenosis is common in older people- a growing and vulnerable population. ${ }^{2}$

(4) Facilitate patient access to medical intervention.

(5) Educate and incentivise clinicians and patients in implementing current best practice medical intervention. This requires acknowledging that medical intervention is a relatively time-consuming, ongoing and poorly remunerated service. Moreover, it requires acknowledging that medical intervention is the most effective tool for lowering risk of stroke and other arterial disease complications and it is highly effective. ${ }^{2}$

\section{References}

1. Paraskevas KI, Mikhailidis DP, Baradaran H, Davies AH, Eckstein $H H$, Faggioli $G$, et al. Management of patients with asymptomatic carotid stenosis may need to be individualized: a multidisciplinary call for action. J Stroke 2021;23:202-212.

2. Abbott $A L$, Brunser $A M$, Giannoukas $A$, Harbaugh RE, Kleinig $T$, Lattanzi $S$, et al. Misconceptions regarding the adequacy of best medical intervention alone for asymptomatic carotid stenosis. J Vasc Surg 2020;71:257-269.

3. Executive Committee for the Asymptomatic Carotid Atherosclerosis Study. Endarterectomy for asymptomatic carotid artery stenosis. JAMA 1995;273:1421-1428.

4. Halliday $A$, Harrison $M$, Hayter $E$, Kong $X$, Mansfield $A$, Marro $J$, et al. 10-Year stroke prevention after successful carotid endarterectomy for asymptomatic stenosis (ACST-1): a multicentre randomised trial. Lancet 2010;376:1074-1084.

5. Naylor AR, Ricco JB, de Borst GJ, Debus S, de Haro J, Halliday $A$, et al. Editor's choice - Management of atherosclerotic carotid and vertebral artery disease: 2017 clinical practice guidelines of the European Society for Vascular Surgery (ESVS). Eur J Vasc Endovasc Surg 2018;55:3-81.

6. Halliday A, Bulbulia R, Bonati LH, Chester J, Cradduck-Bamford $A$, Peto $R$, et al. Second asymptomatic carotid surgery 
trial (ACST-2): a randomised comparison of carotid artery stenting versus carotid endarterectomy. Lancet 2021;398: 1065-1073.

7. Batchelder AJ, Saratzis A, Ross Naylor A. Editor's choice Overview of primary and secondary analyses from 20 randomised controlled trials comparing carotid artery stenting with carotid endarterectomy. Eur J Vasc Endovasc Surg 2019; 58:479-493.

8. Brott TG, Howard G, Roubin GS, Meschia JF, Mackey A, Brooks $W$, et al. Long-term results of stenting versus endarterectomy for carotid-artery stenosis. N Engl J Med 2016;374:1021-1031.

9. Bonati LH, Dobson J, Featherstone RL, Ederle J, van der Worp $H B$, de Borst GJ, et al. Long-term outcomes after stenting versus endarterectomy for treatment of symptomatic carotid stenosis: the International Carotid Stenting Study (ICSS) ran- domised trial. Lancet 2015;385:529-538.

10. Abbott A. Asymptomatic carotid stenosis and stroke risk. Lancet Neurol 2021;20:698-699.

Correspondence: Anne L. Abbott

Department of Neuroscience, Central Clinical School, Monash University, Level 6, 99 Commercial Rd, Melbourne 3004, Australia

Tel: +61-3-9903-0304

Fax: +61-3-8679-3341

E-mail: Anne.L.Abbott@gmail.com

https://orcid.org/0000-0002-7228-4909

Received: August 30, 2021

Revised: November 1, 2021

Accepted: December 31, 2021

The author has no financial conflicts of interest. 